
\title{
BResarch Soute \\ Research on Space-time Blocking Matrix Algorithm Based on Frequency Diverse Array
}

\author{
Yanping Liao \\ Harbin Engineering University \\ Zenan Wu ( $\nabla$ wuzenan@hrbeu.edu.cn ) \\ Harbin Engineering University
}

\section{Research}

Keywords: frequency diverse array, blocking matrix processing, space-time processing, mainlobe interference

Posted Date: March 11th, 2020

DOI: https://doi.org/10.21203/rs.3.rs-16639/v1

License: (1) This work is licensed under a Creative Commons Attribution 4.0 International License. Read Full License 


\title{
Research on Space-time Blocking Matrix Algorithm Based on Frequency Diverse Array
}

\author{
Yanping Liao, Zenan $\mathrm{Wu}^{*}$
}

\begin{abstract}
Because the beam pattern of the conventional phased array or MIMO radar is only related to the angle dimension, it is difficult to suppress the incident interference when it is in the mainlobe or at the same angle of the desired signal. According to the development demand of mainlobe anti-interference technology. In this paper, a space - time blocking matrix processing algorithm based on FDA is proposed. The algorithm gives the specific structure of the blocking matrix, which can not only avoid the interference of the same angle and different distances, but also eliminate the interference signal in the direction of the mainlobe without affecting the energy of other incident signals, so as to ensure the good shape of the beam pattern. The simulation results show that the algorithm can successfully suppress the mainlobe interference and has good beam-conformal.
\end{abstract}

Index Terms-frequency diverse array; blocking matrix processing; space-time processing; mainlobe interference

\section{INTRODUCTION}

$\mathrm{W}$ ith the development of information technology, the electromagnetic environment in which modern communication and detection equipment are located becomes more and more complex, which puts forward higher requirements for anti-interference technology. Due to its good performance, adaptive beamforming technology has been applied in the field of radar anti-jamming and achieved good result. However, when there is mainlobe interference, the traditional adaptive beamforming will cause serious distortion of the mainlobe and shift [1-2], which will affect its target detection and tracking functions. Therefore, anti-mainlobe interference has become a hot research topic in recent years. Various algorithms have been proposed for mainlobe interference suppression technology. Jablon N K[3] proposed that the solution to the mainlobe deformation is diagonal loading technique, but it is very difficult to determine the loading amount. S. A. vorobyov put forward SOC algorithm [4], and he thought that calculating diagonal loading is equivalent to SOC optimization problem, which can be solved by SeDuMi tool. However, the computational complexity of SOC method is too large, and it cannot give an analytical

This work was supported by the Fundamental Research Funds for the Central Universities.

Yanping Liao and Zenan $\mathrm{Wu}$ are with Information and Communication Engineering Department, Harbin Engineering University, Harbin150001, China (e-mail: liaoyanping@hrbeu.edu.cn, wuzenan@hrbeu.edu.cn). algorithm expression. Dai et al. put forward a spatial polarization filtering technology in literature [5]. It obtained the estimation of orthogonal polarization echo and polarization state through the polarization slow variation characteristic of array antenna in spatial domain scanning. Finally, polarization filtering was implemented with optimal polarization to achieve the purpose of suppressing interference. However, this method had no suppression effect when the polarized broadband is different or the mainlobe interference is forwarding interference. Literature [6] proposed a mainlobe interference technique for large aperture auxiliary arrays, but this method requires a lot of space for array placement, increasing the complexity of the system. S. J. YU and J. H. Lee et al. introduced the concept of blocking matrix for the first time in literature [7], and studied a construction method of blocking matrix to solve the problem of mixing the desired signal into the sampled signal. After that, the method was widely applied to the suppression of mainlobe interference. Frost [8] proposed STAP technology for the first time, which can significantly improve the anti-interference performance of the system. The Linear Constrained Minimum Variance (LCMV) algorithm based on Space-Time Adaptive Processing (STAP) has better anti-interference performance [9], because STAP technology can have more degrees of freedom without increasing the number of array elements, thus it can effectively suppress more interference and improve output signal-to-interference-noise ratio of the system [10,11]. Literature [12] proposed the robust constrained inverse beamforming algorithm based on STAP, which can not only reduce the sidelobe level to suppress interference better, but also achieve the lower complexity. The above methods are all researched on the basis of the traditional phased array, and the frequency diverse array(FDA) has a good application prospect in interference suppression due to its excellent beam performance[13]. The angle-distance two-dimensional dependence of the FDA beam pattern makes it naturally have radio frequency stealth characteristics[14]. By designing the weight vector, the mainlobe of the FDA can avoid interference of the same angle and different distances, thus achieving the purpose of interference suppression [15]. However, when the angle and distance of interference are both located in the mainlobe of FDA, anti-mainlobe interference algorithm is still needed. And this algorithm must be effective for both angle and distance at the same time.

Therefore, this paper proposes a space-time blocking matrix processing(BMP) algorithm based on FDA, deduces the space-time steering vector of conventional FDA, and constructs 
its blocking matrix on this basis. However, due to the periodicity of the conventional FDA beam, interference tends to enter from the periodic mainlobe of the beam, resulting in beam shift. In order to solve this problem, this paper proposes a space-time blocking matrix processing algorithm for dot-shape beam, and constructs a space-time blocking matrix on the basis of multi-carrier FDA, which eliminates the periodicity of beam and achieves the purpose of suppressing mainlobe interference.

The paper is organized as follows, Section I is the introduction part of this paper. The Section II mainly introduces proposed the blocking matrix construction method for conventional FDA. The Section III mainly introduces proposed space-time blocking matrix construction method for dot-shape beam. Section IV is the simulation results, and Section V is the conclusion.

\section{PROPOSED STAP-BMP METHOD FOR CONVENTIONAL FDA}

The research of space-time blocking matrix algorithm is based on FDA structure and its related signal model. Therefore, this section mainly introduces its array structure and space-time blocking matrix algorithm.

In Fig.1, $f_{i}$ denotes the transmission frequency of each antenna element, where $i=0,1, \cdots, M-1 . M$ is the number of transmitting elements, $d$ is the spacing of adjacent elements, and the leftmost element is used as the reference point. Then $r$ and $\theta$ respectively represent the distance from the far field observation point to the reference array element and the azimuth angle of the far field observation point relative to the array normal direction. In order to simplify the signal model, in general, only the radiation characteristics of the plane in which the antenna array is located are discussed, and the characteristics in other orientations of the space are not considered.

The biggest feature of the frequency diverse array is its frequency diverse characteristic, that is, a linearly increasing frequency offset between adjacent elements, namely $\Delta f$. The frequency of the signal emitted by the $m$-th array element can be expressed as

$$
f_{m}=f_{0}+m \cdot \Delta f, m=0,1, \cdots, M-1
$$

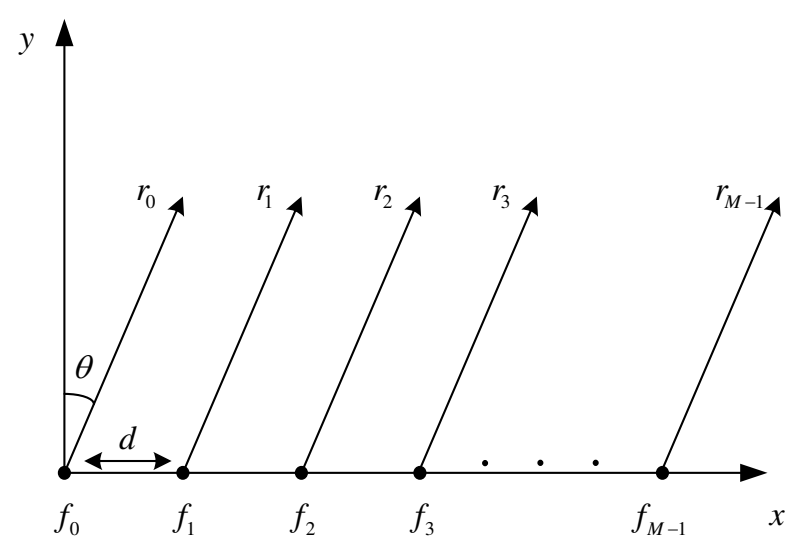

Fig. 1 Uniform linear frequency diverse array(ULA-FDA) structure

Then the array steering vector of the FDA can be expressed as

$$
\begin{aligned}
\boldsymbol{\alpha}(\theta, r)= & {\left[1 \exp \left\{\mathrm{j} 2 \pi\left(\frac{f_{0} d \sin \theta}{c}-\Delta f \frac{r}{c}\right)\right\} \cdots\right.} \\
& \left.\exp \left\{\mathrm{j} 2 \pi(M-1)\left(\frac{f_{0} d \sin \theta}{c}-\Delta f \frac{r}{c}\right)\right\}\right]^{\mathrm{T}}
\end{aligned}
$$

As shown in Fig.2, the basic principle of space-time adaptive processing technology is to apply a plurality of delay units behind each array element of the existing array without enlarging the aperture of the array. From the perspective of array elements, the delay units applied behind each array element are equivalent to time domain filters one by one, which are used to filter out interference signals in time domain. According to the delay unit of each stage, several array elements form an antenna array, which is equivalent to a spatial filtering structure and is used to distinguish interference signals incident in different directions in space.

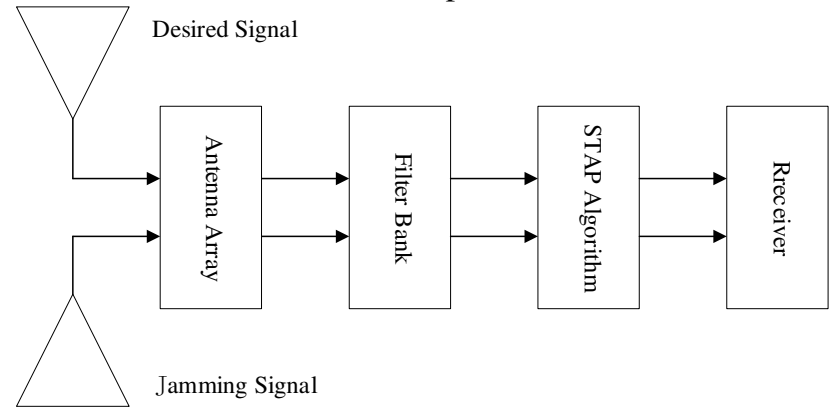

Fig.2 Block diagram of space-time anti-jamming system

After signals and interferences enter the array antenna, filtered by the filter bank. The processed signal is subjected to space-time two-dimensional processing, and finally the processed signal is sent to a receiver. The signal received at the $p$ tap of the $m$-th array element can be expressed as $x_{m}\left(t-(p-1) T_{s}\right),(m=1,2, \cdots, M ; p=1,2, \cdots, P)$.

Then, the optimal weight vector $w_{m p}$ is obtained through an appropriate space-time anti-jamming algorithm in order to suppress interference in the received signal and improve beam performance.

Then the space-time processed output signal $y(t)$ can be expressed as

$$
y(t)=\sum_{m=1}^{M} \sum_{p=1}^{P} w_{m p} x_{m}\left(t-(p-1) T_{s}\right)
$$

Since the fundamental principle of time domain filtering in STAP is to apply several time taps after each array element, these several time taps are equivalent to one FIR filter for time domain filter.

Supposing that the number of time taps is $P$, then the dimension of its STAP is $L=M P$. Considering the structural characteristics of the FDA, the time domain steering vector of the FDA can be expressed as:

$$
\begin{gathered}
\boldsymbol{\alpha}_{t}(\omega)=\left[\boldsymbol{\alpha}_{t 1}(\omega), \boldsymbol{\alpha}_{t 2}(\omega), \ldots, \boldsymbol{\alpha}_{t m}(\omega), \cdots, \boldsymbol{\alpha}_{t M}(\omega)\right]^{\mathrm{T}} \\
\boldsymbol{\alpha}_{t m}(\omega)=\left[1 \exp \left\{\mathrm{j} 2 \pi\left(f_{0}+(m-1) \Delta f\right) T_{s}\right\} \cdots\right. \\
\left.\exp \left\{\mathrm{j} 2 \pi(P-1)\left(f_{0}+(m-1) \Delta f\right) T_{s}\right\}\right]^{\mathrm{T}}
\end{gathered}
$$

where $p=1,2, \cdots, P ; m=1,2, \cdots, M$ 
Then the space-time steering vector of the frequency control array is the Hadamard product of its space-time steering vector and its time-domain steering vector, which can be expressed as

$$
\boldsymbol{\alpha}(\theta, r, \omega)=\boldsymbol{\alpha}(\theta, r) \square \boldsymbol{\alpha}_{t}(\omega)
$$

As shown in Fig.3, the essence of Blocking Matrix Processing (BMP) algorithm is to use the constructed blocking matrix to mask the mainlobe interference in the received signal, and then use the preprocessed received data for space-time processing to achieve the purpose of suppressing the mainlobe interference.

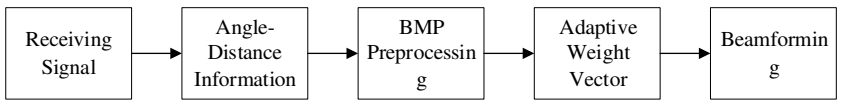

Fig.3 BMP algorithm Process chart

Supposing that the signal received by a uniform linear FDA including a desired signal and $K$ other sidelobe interference with a $i$-th mainlobe interference. From (6), it can be seen that the space-time steering vector of the FDA has both the frequency diversity characteristic brought by frequency offset $\Delta f$ and the delay characteristic brought by time tap $P$. According to the basic idea of adjacent array element cancellation, considering the above two characteristics, meanwhile the angle-distance information of the mainlobe interference is taken as the cancellation factor. We proposed the specific structure of the space-time blocking matrix of the FDA as follows

$$
\mathbf{B}=\left[\begin{array}{cccccc}
1 & -e^{\mathrm{j} \varphi_{m}} & 0 & \cdots & 0 & 0 \\
0 & 1 & -e^{\mathrm{j} \varphi_{m}} & \cdots & 0 & 0 \\
\vdots & \vdots & \vdots & \vdots & \cdots & \vdots \\
0 & 0 & e^{\mathrm{j}(p-1) \gamma_{i m}} & -e^{\mathrm{j} \mu_{i}} & \cdots & 0 \\
\vdots & \vdots & \vdots & \vdots & \cdots & \vdots \\
0 & 0 & \cdots & 1 & -e^{\mathrm{j} \varphi_{m}} & 0 \\
0 & 0 & \cdots & 0 & 1 & -e^{\mathrm{j} \varphi_{m}}
\end{array}\right]_{(L-1) \times L}
$$

where $\quad L=M P \quad, \quad \varphi_{m}=\gamma_{i m}=2 \pi T_{s}\left(f_{0}+(m-1) \Delta f\right)$ ,$\mu_{i}=2 \pi T_{s}\left(f_{0} d \frac{\sin \theta_{i}}{c}-\Delta f \frac{r_{i}}{c}\right)$. In matrix $\mathbf{B}, e^{\mathrm{j}(p-1) \gamma_{i m}}$ and $-e^{\mathrm{j} \mu_{i}}$ are located at the position of $(m P, m P)$ and $(m P, m P+1)$ of the matrix respectively.

Before the signal is preprocessed, the signal on the $p$ tap of the $m$ array element can be written as follows:

$$
\begin{aligned}
x_{m}\left(t-(p-1) T_{s}\right)= & \sum_{k=0}^{K+1} s_{k}\left(t-(p-1) T_{s}\right) e^{-\mathrm{j}\left[(p-1) \gamma_{k m}+(m-1) \mu_{k}\right]} \\
& +n\left(t-(p-1) T_{s}\right)
\end{aligned}
$$

The signal processed by the BMP can be expressed as

$$
\begin{aligned}
& \hat{x_{m}}\left(t-(p-1) T_{s}\right)=\sum_{k=0}^{K+1} \hat{s_{k}}\left(t-(p-1) T_{s}\right) e^{\left.-\mathrm{ji}(p-1) \gamma_{k m}+(m-1) \mu_{k}\right]} \\
& +\hat{n}\left(t-(p-1) T_{s}\right) \\
& \hat{s_{k}}\left(t-(p-1) T_{s}\right)=\left\{\begin{array}{l}
s_{k}\left(t-(p-1) T_{s}\right)\left[1-e^{\mathrm{j}\left(\gamma_{k m}-\varphi_{m}\right)}\right] \\
(p \neq P \text { 且 } m p \neq M P) \\
s_{k}\left(t-(p-1) T_{s}\right)\left[e^{\mathrm{j}(p-1) \gamma_{\gamma_{m}}}-e^{\mathrm{j}(p-1) \gamma_{i m}} e^{\mathrm{j}\left(\mu_{k}-\mu_{k}\right)}\right] \\
(p=P)
\end{array}\right.
\end{aligned}
$$

\section{PROPOSED STAP-BMP METHOD FOR DOT-SHAPE BEAM}

When the sidelobe interference is located at other energy peaks of the s-beam of the FDA, it will form null steering while affecting the performance of the beam. This is caused by the self-characteristics of the conventional FDA. Therefore, it is important to form dot-shape beam for mainlobe interference suppression.

Multi-carrier is based on frequency diverse array, that is, each array element no longer transmits the traditional single carrier signal, but transmits multi-carrier signals with different frequencies. The structure diagram of the multi-carrier frequency diverse array system is shown in the Fig.4.

The intermediate array element of the line array is used as the reference array element, and the two sides of the reference array element are set as symmetric frequency structure, and the symmetry benefits can make the energy more concentrated. Then the $n$th subcarrier of the $m$ th array element can be expressed as:

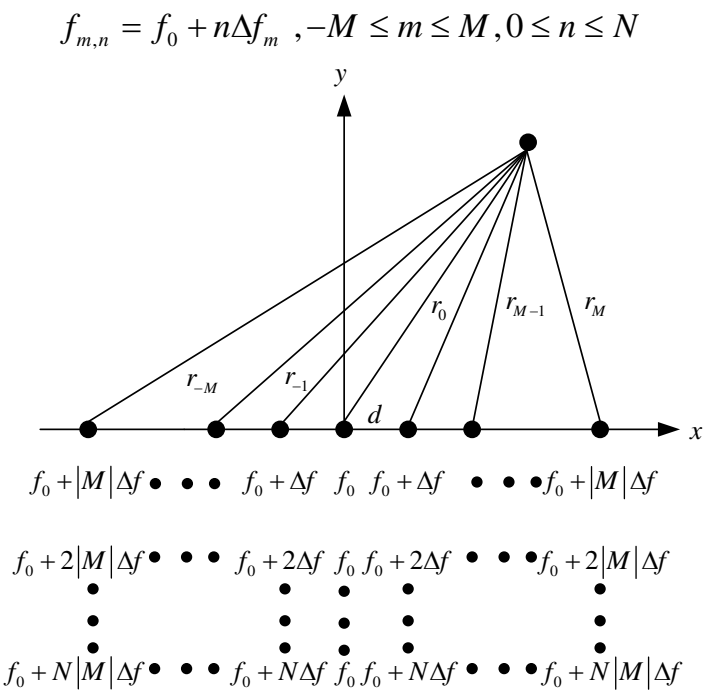

Fig. 4 Multi-carrier FDA model

where $N$ represents the number of subcarriers and $M$ represents the number of elements, $\Delta f_{m}$ is a frequency offset scheme we proposed consisting of a square root function, which can be expressed as:

$$
\Delta f_{m}=\Delta \delta \cdot|m|^{\frac{1}{2}}, \quad-M \leq m \leq M
$$

where $\Delta \delta$ is a small frequency offset constant. The square root frequency offset based on the multi-carrier FDA we proposed can make the beam energy more concentrated, thus forming a dot-shape beam. Then the array steering vector of the multi-carrier FDA can be expressed as

$$
\begin{aligned}
\boldsymbol{\beta}(\theta, r)= & {\left[\boldsymbol{\beta}_{-M 1}(\theta, r), \cdots \boldsymbol{\beta}_{-M n}(\theta, r), \ldots, \boldsymbol{\beta}_{-M N}(\theta, r), \ldots\right.} \\
& \left.\boldsymbol{\beta}_{M 1}(\theta, r), \cdots, \boldsymbol{\beta}_{M n}(\theta, r), \ldots, \boldsymbol{\beta}_{M N}(\theta, r)\right]^{\mathrm{T}}
\end{aligned}
$$

where $\boldsymbol{\beta}_{m n}(\theta, r)=\exp \left\{\mathrm{j} 2 \pi\left(\frac{f_{0} m d \sin \theta}{c}-n|m|^{\frac{1}{2}} \Delta f \frac{r}{c}\right)\right\}$

Using the time domain filtering principle of STAP, when the number of time taps is $P$, its dimension is $L=M N P$, then the time domain steering vector of the multi-carrier FDA can be expressed as 


$$
\begin{gathered}
(\omega)=\bigcap_{{ }_{-M) 1}}(\omega), \ldots P_{-M) \mathrm{N}}(\omega), \ldots \\
\left.\bigcap_{M}(\omega), \ldots \bigcap_{M N}(\omega)\right]^{\mathrm{T}}
\end{gathered}
$$

where $(\omega)=\left[1 \exp \left\{\mathrm{j} 2 \pi\left(f_{0}+n|m|^{\frac{1}{2}} \Delta f\right) T_{s}\right\} \cdots\right.$

$$
\left.\exp \left\{\mathrm{j} 2 \pi(P-1)\left(f_{0}+n|m|^{\frac{1}{2}} \Delta f\right) T_{s}\right\}\right]^{\mathrm{T}}
$$

Then the space-time steering vector of the multi-carrier FDA can be expressed as

$$
\theta, r, \omega)=\bigcap \theta, r) \square(\omega)
$$

It can be seen from (14) that the multi-carrier FDA adds one dimension while forming dot-shape beam. This will make the construction of blocking matrix more complicated. According to the basic idea of adjacent array element cancellation, considering the square root frequency offset scheme $f_{m, n}$ and time delay characteristics of multi-carrier FDA, meanwhile the angle-distance information of the mainlobe interference is taken as the cancellation factor. We proposed the specific structure of the space-time blocking matrix of the dot-shape beam as follows:

$\mathbf{B}=\left[\begin{array}{cccccc}1 & -e^{\mathrm{j} u_{m n}} & 0 & \cdots & 0 & 0 \\ 0 & 1 & -e^{\mathrm{j} u_{m n}} & \cdots & 0 & 0 \\ \vdots & \vdots & \vdots & \vdots & \vdots & \vdots \\ 0 & 0 & e^{\mathrm{j}(p-1) \eta_{i m n}} & -e^{\mathrm{j} q_{m}} & \cdots & 0 \\ \vdots & \vdots & \vdots & \vdots & \cdots & \vdots \\ 0 & 0 & 0 & e^{\mathrm{j}(N-1) h_{m}} e^{\mathrm{j}(p-1) \eta_{i m n}} & -e^{\mathrm{j} v_{i}} & 0 \\ \vdots & \vdots & \vdots & \vdots & \cdots & \vdots \\ 0 & 0 & \cdots & 1 & -e^{\mathrm{j} u_{m n}} & 0 \\ 0 & 0 & \cdots & 0 & 1 & -e^{\mathrm{j} u_{m n}}\end{array}\right]_{(L-1) \times L}$

where $L=M N P \quad, \quad u_{m n}=\eta_{i m n}=2 \pi T_{s}\left(f_{0}+n|m|^{\frac{1}{2}} \Delta f\right)$

$q_{m}=h_{m}=2 \pi\left(-|m| \frac{1}{2} \Delta f \frac{r_{1}}{c}\right) \quad, \quad v_{i}=2 \pi\left(f_{0} d \sin \theta_{i}\right)$

matrix $\mathbf{B}, e^{\mathrm{j}(p-1) \eta_{i m n}}$ and $-e^{\mathrm{j} q_{m}}$ are located at the positions of $\quad\left(\left(m+\frac{M-1}{2}\right) n P,\left(m+\frac{M-1}{2}\right) n P\right)$ and $\left(\left(m+\frac{M-1}{2}\right) n P,\left(m+\frac{M-1}{2}\right) n P+1\right)$ of the matrix respectively with $n \neq N \cdot e^{\mathrm{j}(p-1) \eta_{i m n}}$ 与 $-e^{\mathrm{j} h_{m n}}$ are located at the position of $\quad\left(\left(m+\frac{M-1}{2}+1\right) N P,\left(m+\frac{M-1}{2}+1\right) N P\right)$ and $\left(\left(m+\frac{M-1}{2}+1\right) N P,\left(m+\frac{M-1}{2}+1\right) N P+1\right)$ of the matrix respectively.

Before preprocessing, the signal on the $p$ tap of the $n$ subcarrier of the $m$ array element can be expressed as:

$$
\begin{aligned}
x_{m n}\left(t-(p-1) T_{s}\right)= & \sum_{k=0}^{K+1} s_{k}\left(t-(p-1) T_{s}\right) e^{-\mathrm{j}\left[(p-1) \eta_{k m n}+(n-1)\left(h_{k m}+v_{k}\right)\right]} \\
& +n\left(t-(p-1) T_{s}\right)
\end{aligned}
$$

where $p=1,2, \cdots, P ; m=1,2, \cdots, M$.

The signal processed by the blocking matrix can be expressed as

$$
\begin{aligned}
\hat{x_{m}}\left(t-(p-1) T_{s}\right)= & \sum_{k=0}^{K+1} \hat{s_{k}}\left(t-(p-1) T_{s}\right) e^{-\mathrm{j}\left[(p-1) \eta_{k m n}+(n-1)\left(h_{k m}+v_{k}\right)\right]} \\
& +\hat{n}\left(t-(p-1) T_{s}\right) \\
\hat{s_{k}}\left(t-(p-1) T_{s}\right)= & \left\{\begin{array}{l}
s_{k}\left(t-(p-1) T_{s}\right)\left[1-e^{\mathrm{j}\left(\eta_{k m n}-u_{m n}\right)}\right] \\
(p \neq P \text { 且 } p n \neq N P) \\
s_{k}\left(t-(p-1) T_{s}\right)\left[e^{\mathrm{j}(p-1) \eta_{i m n}}-e^{\mathrm{j}(p-1) \eta_{i m n}} e^{\mathrm{j}\left(h_{k m}-h_{i m}\right)}\right] \\
(p=P) \\
s_{k}\left(t-(p-1) T_{s}\right)\left[e^{\mathrm{j} v_{i}} e^{\mathrm{j}(P-1) \eta_{i m n}}-e^{\mathrm{j} v_{i}} e^{\mathrm{j}(P-1) \eta_{i m n}} e^{\mathrm{j}\left(v_{k}-v_{i}\right)}\right] \\
(p n=N P)
\end{array}\right.
\end{aligned}
$$

It can be seen from (19) that when the mainlobe interference is processed, namely $k=i, \hat{s}\left(t-(p-1) T_{s}\right)=0$ can be obtained, which indicates that the interference at the position of the mainlobe is suppressed by the BMP algorithm, meanwhile when other signals are processed, the BMP algorithm only changes its complex envelope and has no influence on the angle and distance information of the signal in space.

Therefore, it is still necessary to use other sidelobe anti-jamming algorithm to suppress sidelobe interference after the signal is preprocessed by BMP algorithm. In this paper, LCMV algorithm is selected to suppress sidelobe interference. The specific process is as follows:

(1) Firstly, the received signal is preprocessed through the blocking matrix to eliminate the mainlobe interference in the signal. Where $\boldsymbol{X}$ represents the received signal, $\boldsymbol{Y}$ represents the received signal after eliminating the mainlobe interference through BMP, and $\mathbf{R}_{y}$ represents the covariance matrix of the received signal after BMP.

$$
\begin{gathered}
\boldsymbol{Y}=\mathbf{B} \boldsymbol{X} \\
\mathbf{R}_{y}=E\left[\boldsymbol{Y} \boldsymbol{Y}^{H}\right]=\mathbf{B} \mathbf{R}_{x} \mathbf{B}^{H}
\end{gathered}
$$

(2) Using the LCMV Criteria, the optimal solution $\boldsymbol{W}_{\text {opt }}$ of the weight vector can be expressed as

$$
\boldsymbol{W}_{\text {opt }}=\mathbf{R}_{y}^{-1} \mathbf{C}^{H}\left(\mathbf{C}^{H} \mathbf{R}_{y}^{-1} \mathbf{C}\right)^{-1} \boldsymbol{g}^{H}
$$

(3) Finally, the beam pattern expression of the output signal can be expressed as

$$
\boldsymbol{B}(\theta, r)=\boldsymbol{W}_{\text {oq }}^{\mathrm{H}}(\theta, r)
$$

\section{RESULTS AND DISCUSSION}

The simulation parameters in this paper are shown in the following Table I.

TABLE I

THE SIMULATION CONDITION

\begin{tabular}{cc}
\hline \hline Parameters & Value \\
\hline $\begin{array}{c}\text { Number of } \\
\text { array elements } \\
\text { Number of }\end{array}$ & $M=11$ \\
& $N=8$
\end{tabular}

subcarriers 


$\begin{array}{cc}\begin{array}{c}\text { Number of taps } \\ \text { Interval of } \\ \text { array elements } \\ \text { carrier }\end{array} & \begin{array}{c}P=4 \\ d=\lambda / 2\end{array} \\ \text { frequency } & f_{0}=10 \mathrm{GHz} \\ \text { Desired signal } & \left(\theta_{0}, r_{0}\right)=\left(40^{\circ}, 120 \mathrm{~km}\right) \\ \text { Main lobe } & \left(\theta_{1}, r_{1}\right)=\left(40^{\circ}, 120 \mathrm{~km}\right) \\ \text { interference } & \\ \text { signal } & \left(\theta_{2}, r_{2}\right)=\left(10^{\circ}, 60 \mathrm{~km}\right) \\ & \left(\theta_{3}, r_{3}\right)=\left(80^{\circ}, 170 \mathrm{~km}\right) \\ \text { Interference } & -20 \mathrm{~dB} \\ \text { signal } & 40 \mathrm{~dB} \\ \text { SNR } & \\ \text { INR } & \end{array}$

Fig.5 shows the beam pattern under the BMP algorithm. It can be seen from the cursor data that the beam forms the energy peak at the position of the desired signal, and deep null steering can be formed at both sidelobe interference positions, with depths of $-53.78 \mathrm{~dB}$ and $-59.28 \mathrm{~dB}$, respectively.

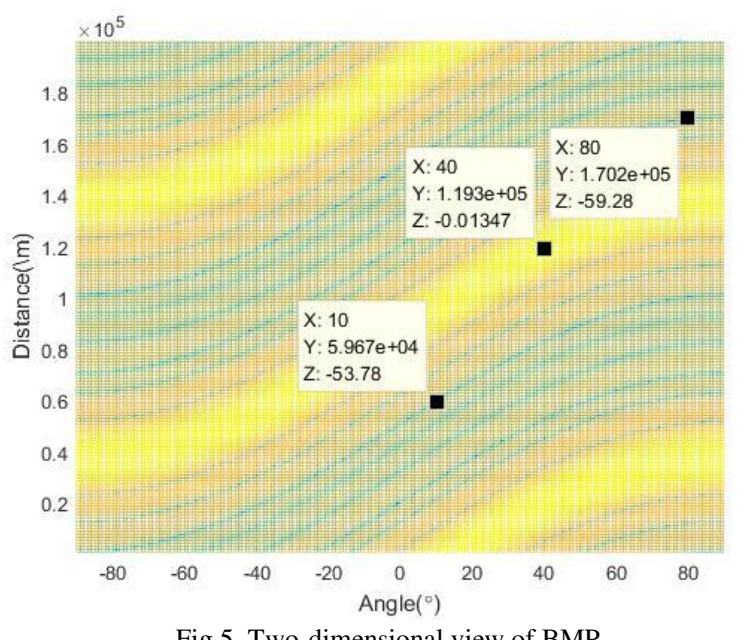

Fig.6 and Fig.7 respectively show the beam pattern of angle dimension and distance dimension at the mainlobe section. As can be seen from the red dotted line in the figure, the LCMV algorithm forms a null steering on the angle and distance of mainlobe interference. As can be seen from the solid blue line in the figure, after preprocessing with the BMP, the interference of the mainlobe of the signal is completely suppressed, and the mainlobe of the beam points to the desired position of the signal. Meanwhile the shape conformation of the beam is very good, which verifies the correctness of the algorithm.

One of the sidelobe interferences is set on the other peaks of the s-beam, that is $\left(\theta_{2}, r_{2}\right)=\left(-20^{\circ}, 80 \mathrm{~km}\right)$, and the resulting beam pattern simulation results are shown in Fig.8. It is not difficult to see from Fig. 8 that when sidelobe interference is set on other peaks of the s-beam, nulls will be formed at the interference position, resulting in shift of the s-beam. this is because sidelobe interference happens at other energy peaks of the s-beam of the FDA, which affects the performance of the beam while forming nulls.

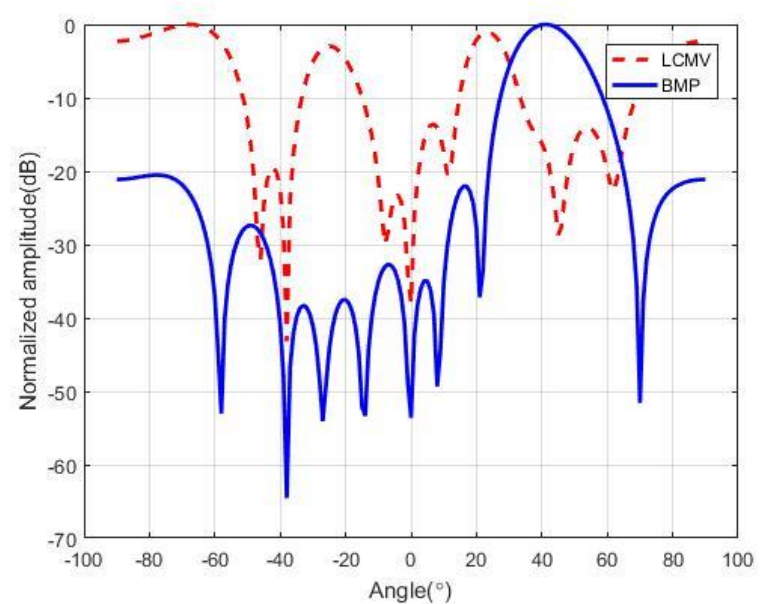

Fig.6. Angle is $40^{\circ}$

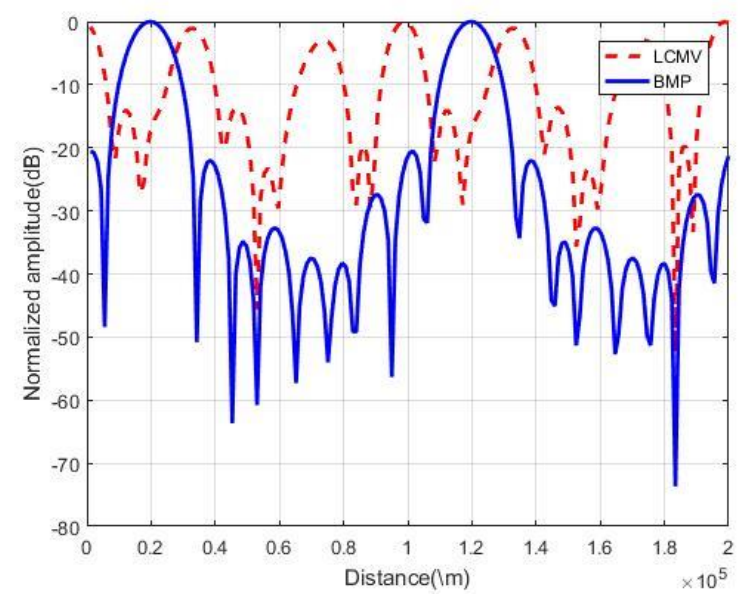

Fig.7. Distance is $120 \mathrm{~km}$

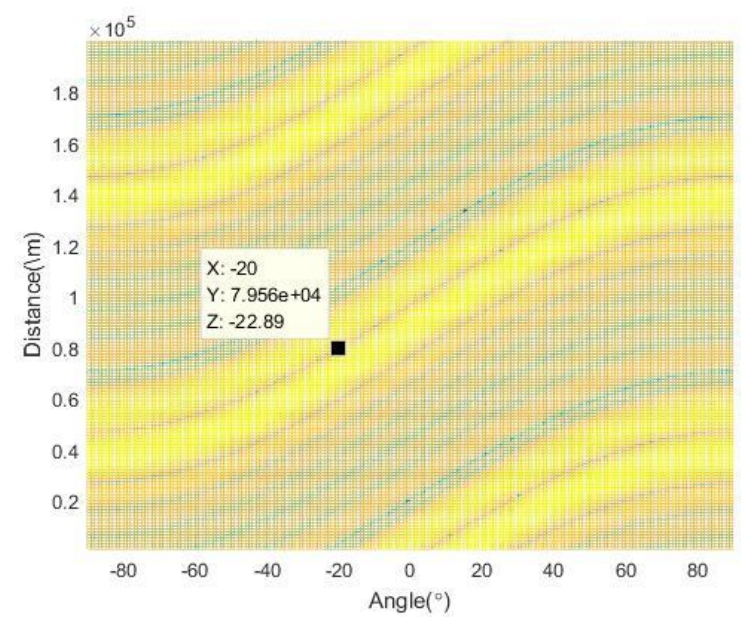

Fig.8. Two-dimensional view of BMP

Fig.9 shows the dot-shape beam pattern under the blocking matrix processing algorithm. As can be seen from the figure, an obvious dot-shape beam can be obtained under this method, and the cursor data reflects that this algorithm can form a deep null steering at both interference positions, with the null steering depths of $-40.79 \mathrm{~dB}$ and $-44.88 \mathrm{~dB}$ respectively.

Fig. 10 and Fig. 11 show the beam pattern of Angle dimension and distance dimension at the mainlobe section, respectively. As can be seen from the red dotted line in the figure, the LCMV algorithm forms null steering in the angle and distance of the 
mainlobe interference. And the solid blue line shows that after preprocessing with the BMP, the mainlobe interference of the signal is completely suppressed, and the shape conformal of the beam is very good, which verifies the correctness of the algorithm. The anti-interference method of the dot-shape beam also eliminates the periodicity of the distance dimension of the s-beam, which solves the problem that the interference signal falls on other energy peaks of the beam.

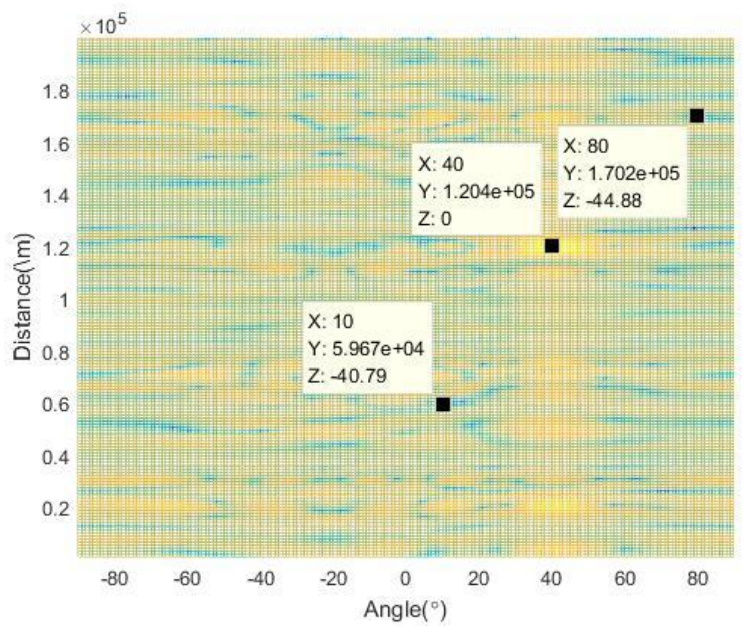

Fig.9. Dot-shape beam of BMP

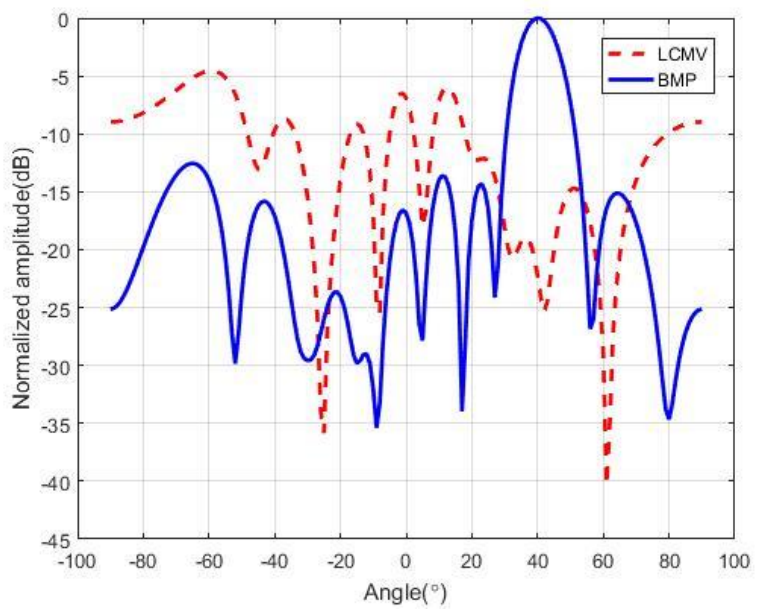

Fig.10. Angle is $40^{\circ}$

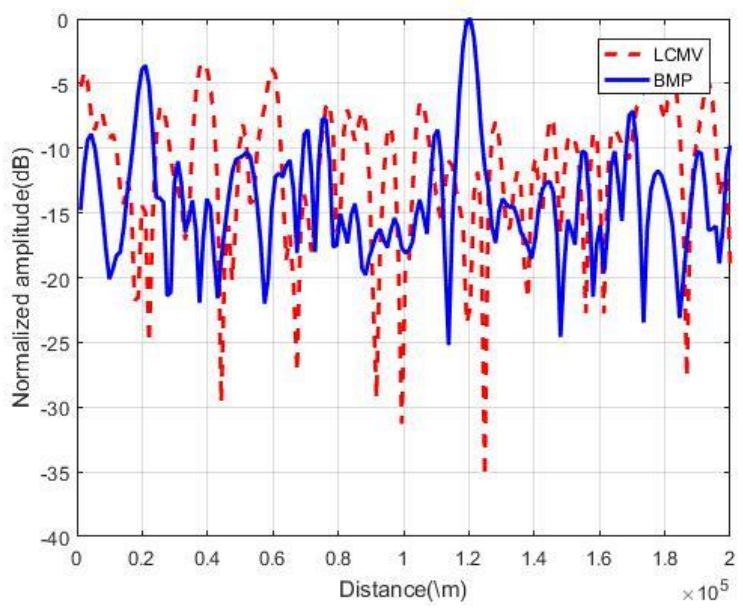

Fig.11. Distance is $120 \mathrm{~km}$

\section{CONCLUSION}

In this paper, a space - time mainlobe interference suppression algorithm based on FDA is proposed. Firstly, the angle - distance space-time vector is derived based on the array factor and space - time model. Secondly, the blocking matrix of the FDA is derived from its space-time steering vector. Finally, aiming at other energy peaks and beam periodicity problems of sidelobe interference located in s-beam, the blocking matrix of dot-shape beam is derived based on multi-carrier FDA to improve the convergence performance of beam energy and eliminate the periodicity of beam, and the interference of main lobe can be well suppressed. At the last, the effectiveness of the proposed algorithm is verified by simulation.

\section{Abbreviations}

FDA: Frequency Diverse Array; STAP: Space-Time Adaptive Processing; BMP: Blocking Matrix Processing; LCMV: Linear Constrained Minimum Variance; ULA-FDA: Uniform Linear Frequency Diverse Array; SNR: Signal-to-Noise Ratio; INR: Interfere-to-Noise Ratio

\section{Authors' contributions}

$\mathrm{ZW}$ proposed the algorithm and developed the mathematical derivations as well as the majority of simulation results. YL helped to comb algorithm principle and check the simulation codes. All authors read and approved the final manuscript.

\section{Acknowledgements}

The authors are grateful to the anonymous referees for their valuable comments and suggestions that improved this paper.

\section{Funding}

This work was supported by the Fundamental Research Funds for the Central Universities.

\section{Availability of data and materials}

Please contact the authors for data requests.

\section{Competing interests}

The authors declare that they have no competing interests.

\section{Author details}

Yanping Liao and Zenan $\mathrm{Wu}$ are with Information and Communication Engineering Department, Harbin Engineering University, Harbin 150001, China (e-mail: liaoyanping@hrbeu.edu.cn,wuzenan@hrbeu.edu.cn).

\section{REFERENCES}

[1] Vendik O G , Kozlov D S . Phased Antenna Array With a Sidelobe Cancellation for Suppression of Jamming[J]. IEEE Antennas and Wireless Propagation Letters, 2012, 11:648-650..

[2] LI,STOCICA P. Robust Adaptive Beamforming [M].New York,USA:Wiley,2005.

[3] Jablon, N. Adaptive beamforming with the generalized sidelobe canceller in the presence of array imperfections[J]. IEEE Transactions on Antennas and Propagation, 1986, 34(8):996-1012. 
[4] Vorobyov S A, Gershman A B , Luo Z Q . Robust adaptive beamforming using worst-case performance optimization: a solution to the signal mismatch problem[J]. IEEE Transactions on Signal Processing, 2003, 51(2):313-324.

[5] Dai H, Wang X, Li Y, et al. Main-Lobe Jamming Suppression Method of using Spatial Polarization Characteristics of Antennas[J]. IEEE Transactions on Aerospace and Electronic Systems, 2012, 48(3):2167---2179.

[6] Xiaopeng Yang, Pilei Yin, Tao Zeng. Mainlobe interference suppression based on large aperture auxiliary array[C]// 2012 IEEE Asia-Pacific Conference on Antennas and Propagation. IEEE, 2012.

[7] Yu S J , Lee J H . Efficient eigenspace-based array signal processing using multiple shift-invariant subarrays[J]. IEEE Transactions on Antennas and Propagation, 1999, 47(1):186-194.

[8] Z. Huang, "Research and implementation of space-time two-dimensional anti-jamming algorithm for Beidou receiver," University of Electronic Science and Technology of China, 2014.

[9] Jianjie Y E. Application of an Improved LCMV Algorithm in GPS Anti-Jamming. Electronic Science \& Technology, 2013.

[10] Liu Y T, Zhang S Y, Shi D, et al. Anti-Jamming Space-Time Processor with Digital Beamforming for Satellite Navigation. Environmental Electromagnetics. IEEE, 2016:31-35.

[11] Lee K, Lee J. Design and Evaluation of Symmetric Space-Time Adaptive Processing of an Array Antenna for Precise Global Navigation Satellite System Receivers. Iet Signal Processing, 2017, 11(6):758-764.

[12] Yanping Liao, Xing Wu, Zenan Wu, et al. Robust Constrained Inverse Beamforming Algorithm Based on Space Time Adaptive Processing[J]. IEEE Access, 2019, 7:55191-55198.

[13] Basit A , Khan W, Khan S, et al. Development of Frequency Diverse Array Radar Technology: A Review[J]. IET Radar Sonar Navigation, 2017, 12(2):165-175.

[14] Abdalla A , Abdalla H , Ramadan M, et al. Overview of frequency diverse array in radar ECCM applications[C]// 2017 International Conference on Communication, Control, Computing and Electronics Engineering (ICCCCEE). IEEE, 2017.

[15] J. Xu, G. Liao, S. Zhu, et al. Deceptive jamming suppression with frequency diverse MIMO radar[J]. Signal Processing, 2015, 113(C):9-17. 
Figures

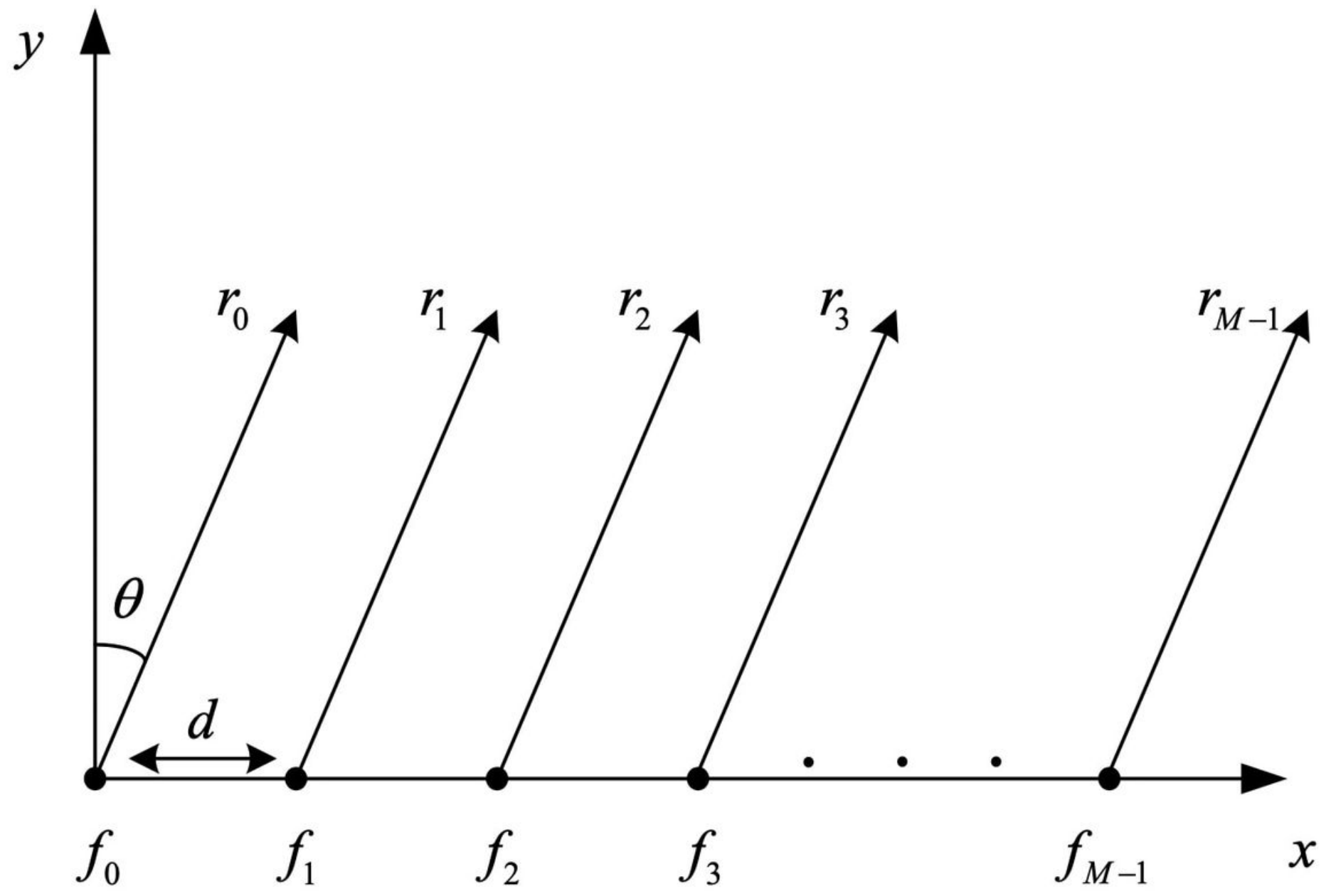

Figure 1

Uniform linear frequency diverse array(ULA-FDA) structure 


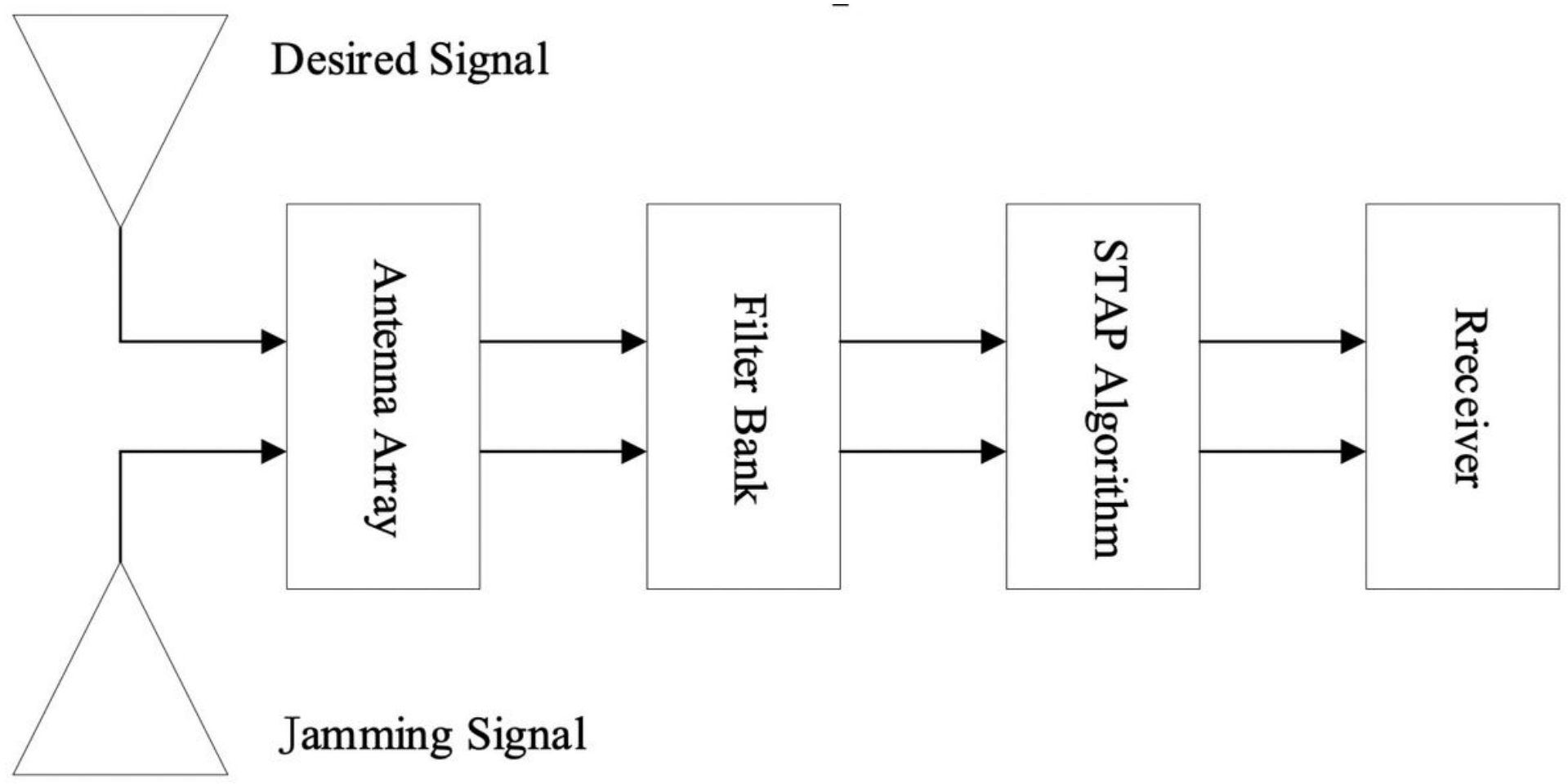

Figure 2

Block diagram of space-time anti-jamming system

\begin{tabular}{|c|c|c|c|c|}
\hline $\begin{array}{c}\text { Receiving } \\
\text { Signal }\end{array}$ & $\begin{array}{c}\text { Angle- } \\
\text { Distance } \\
\text { Information }\end{array}$ & $\begin{array}{c}\text { BMP } \\
\text { Preprocessin } \\
\text { g }\end{array}$ & $\begin{array}{c}\text { Adaptive } \\
\text { Weight } \\
\text { Vector }\end{array}$ & $\begin{array}{c}\text { Beamformin } \\
\mathrm{g}\end{array}$ \\
\hline
\end{tabular}

Figure 3

BMP algorithm Process chart 


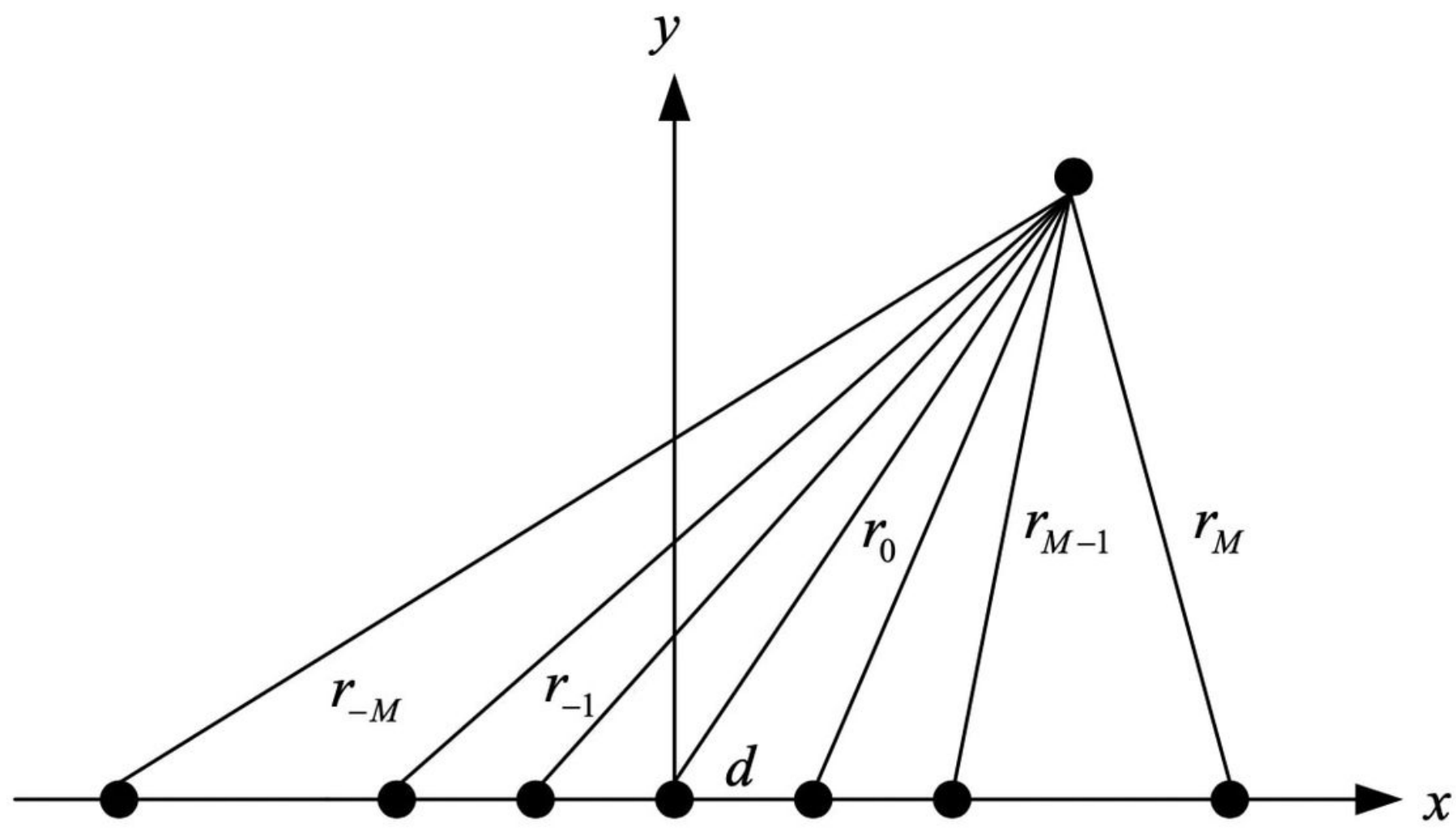

$f_{0}+|M| \Delta f \bullet \bullet f_{0}+\Delta f \quad f_{0} f_{0}+\Delta f \quad \bullet f_{0}+|M| \Delta f$

$f_{0}+2|M| \Delta f \bullet \bullet f_{0}+2 \Delta f f_{0} f_{0}+2 \Delta f \bullet \bullet \bullet f_{0}+2|M| \Delta f$

$f_{0}+N|\stackrel{\bullet}{M}| \Delta f \bullet \bullet f_{0}+\stackrel{\bullet}{N} \Delta f \dot{\bullet}_{0} f_{0}+\stackrel{\bullet}{N} \Delta f \bullet \bullet f_{0}+\stackrel{\bullet}{N}|M| \Delta f$

Figure 4

Multi-carrier FDA model 


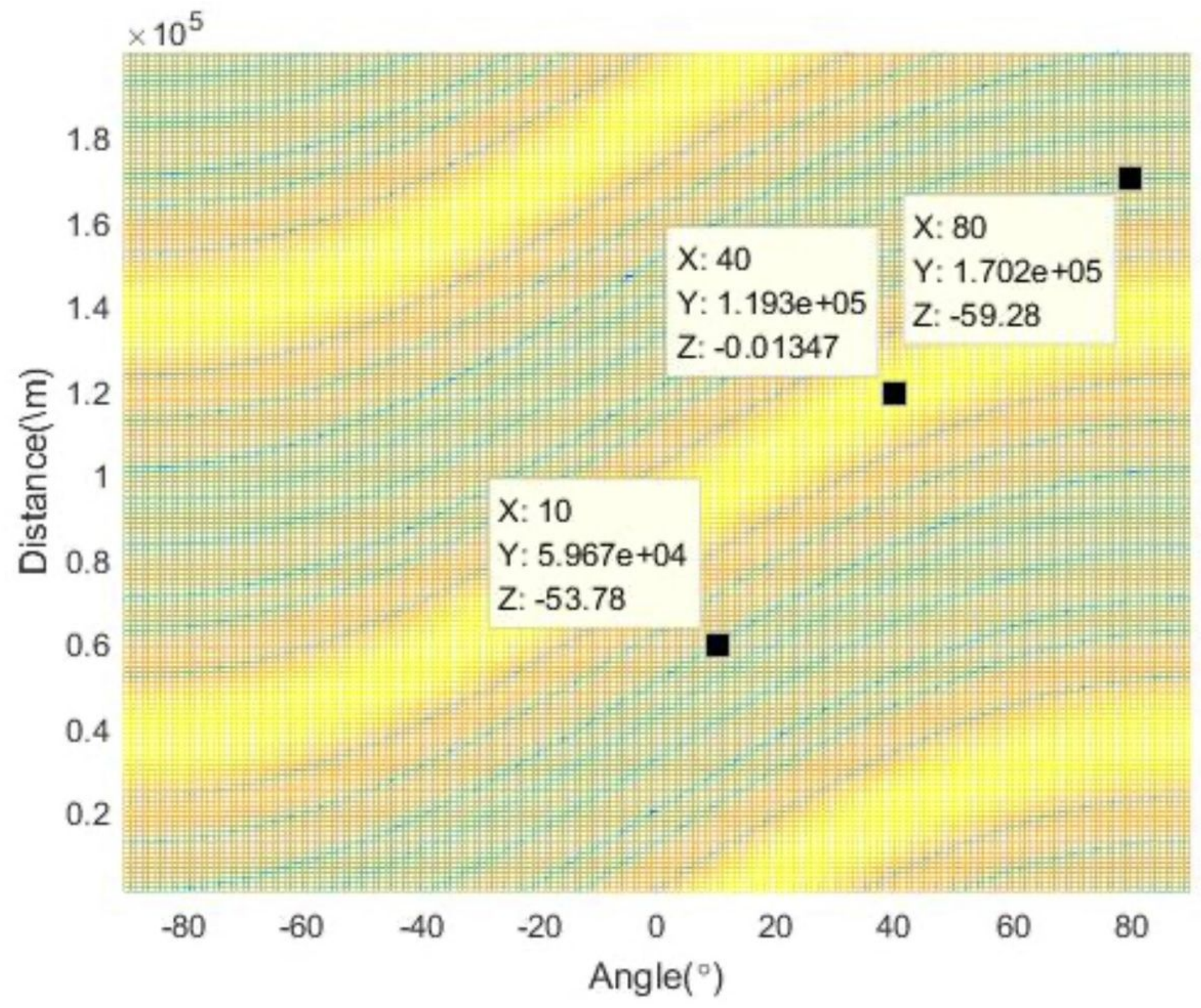

Figure 5

Two-dimensional view of BMP 


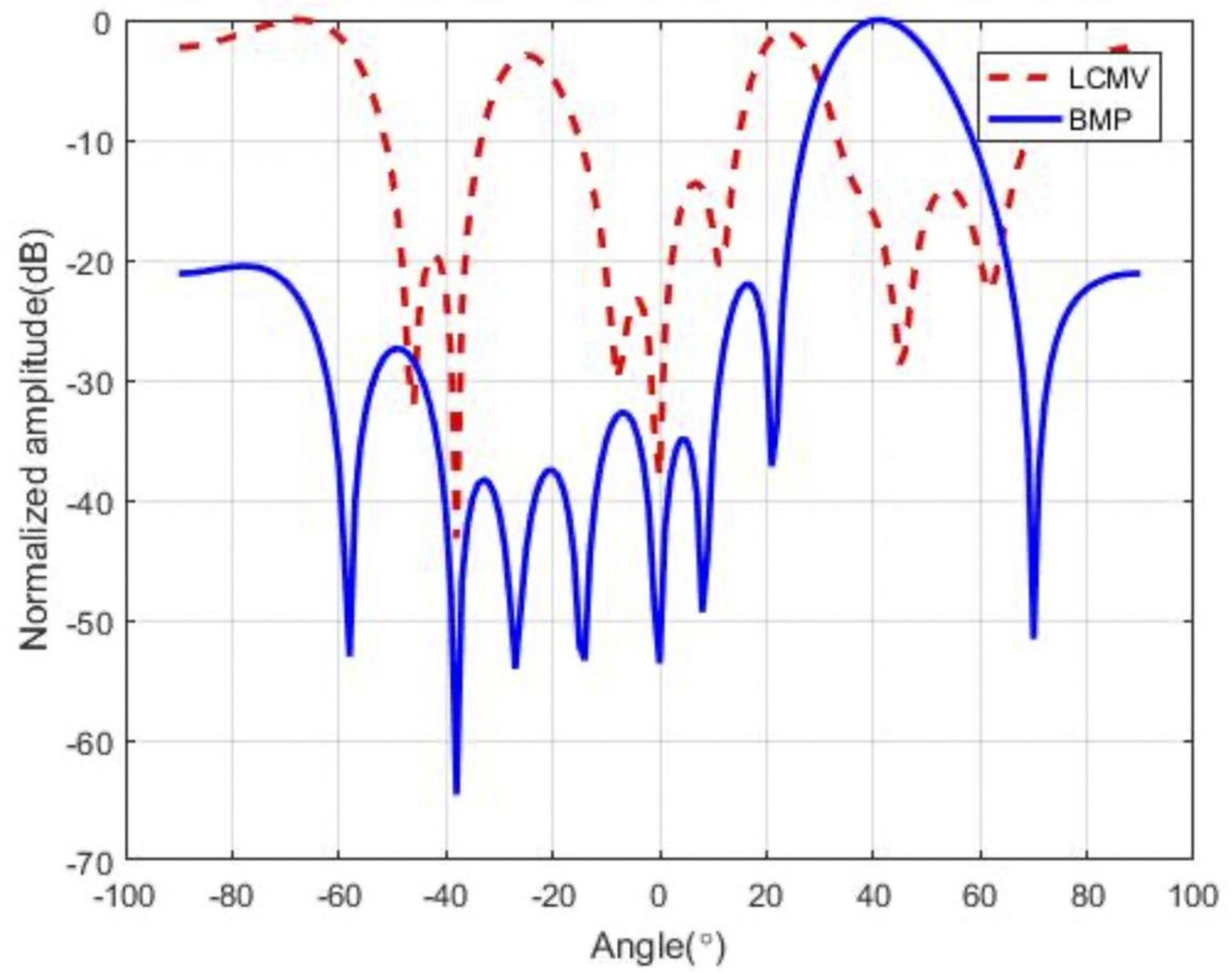

Figure 6

Angle is $40^{\circ}$ 


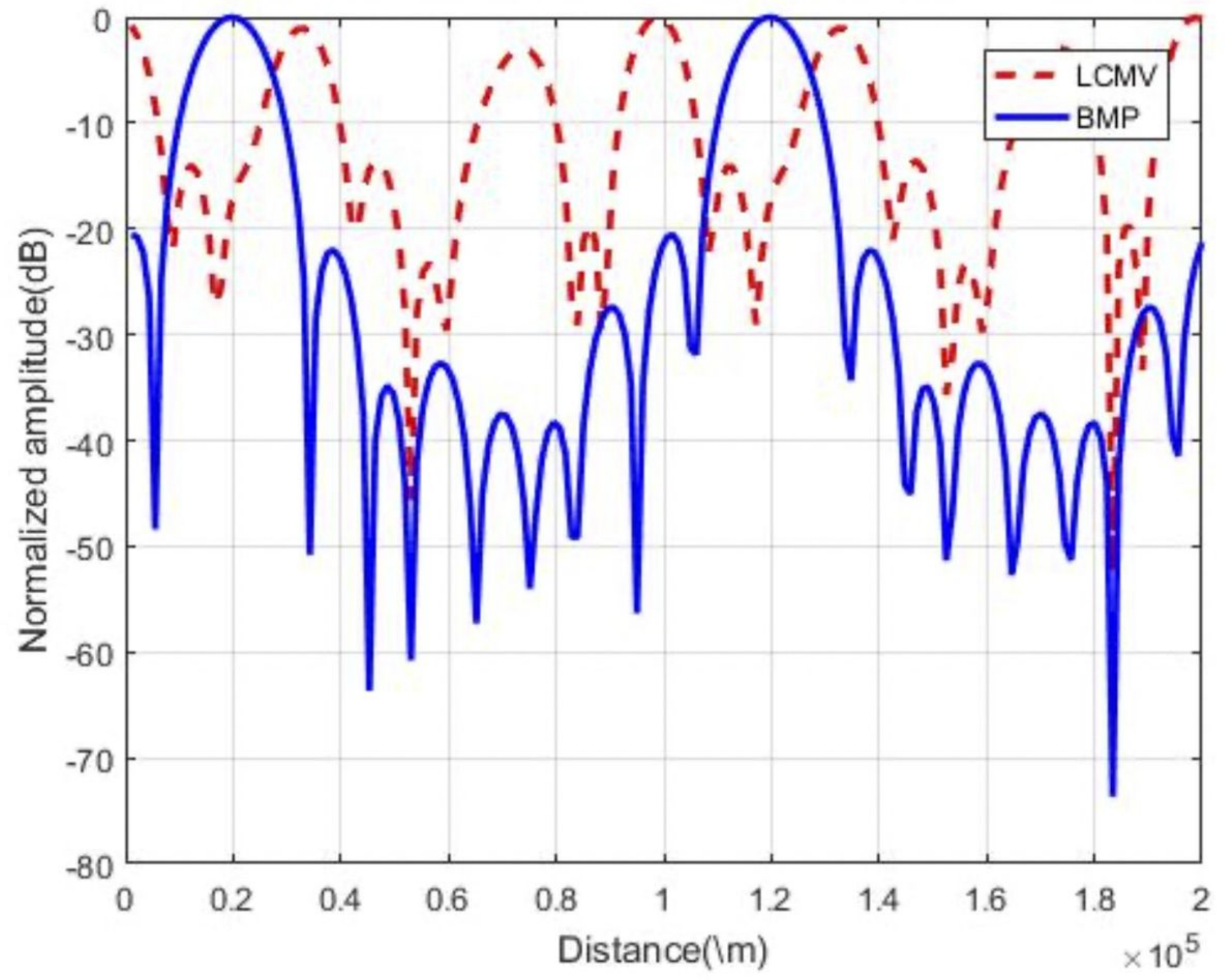

Figure 7

Distance is $120 \mathrm{~km}$ 


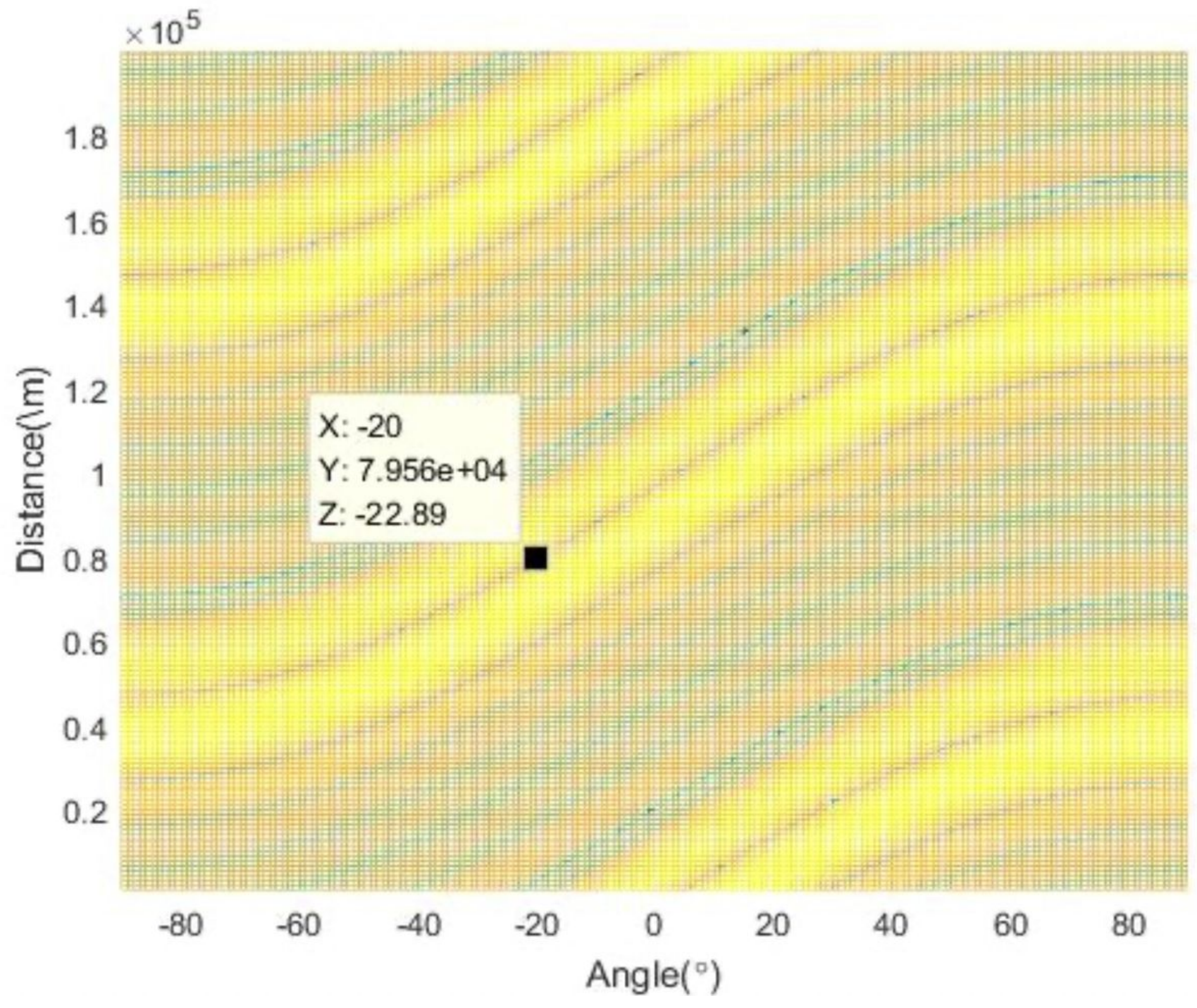

Figure 8

Two-dimensional view of BMP 


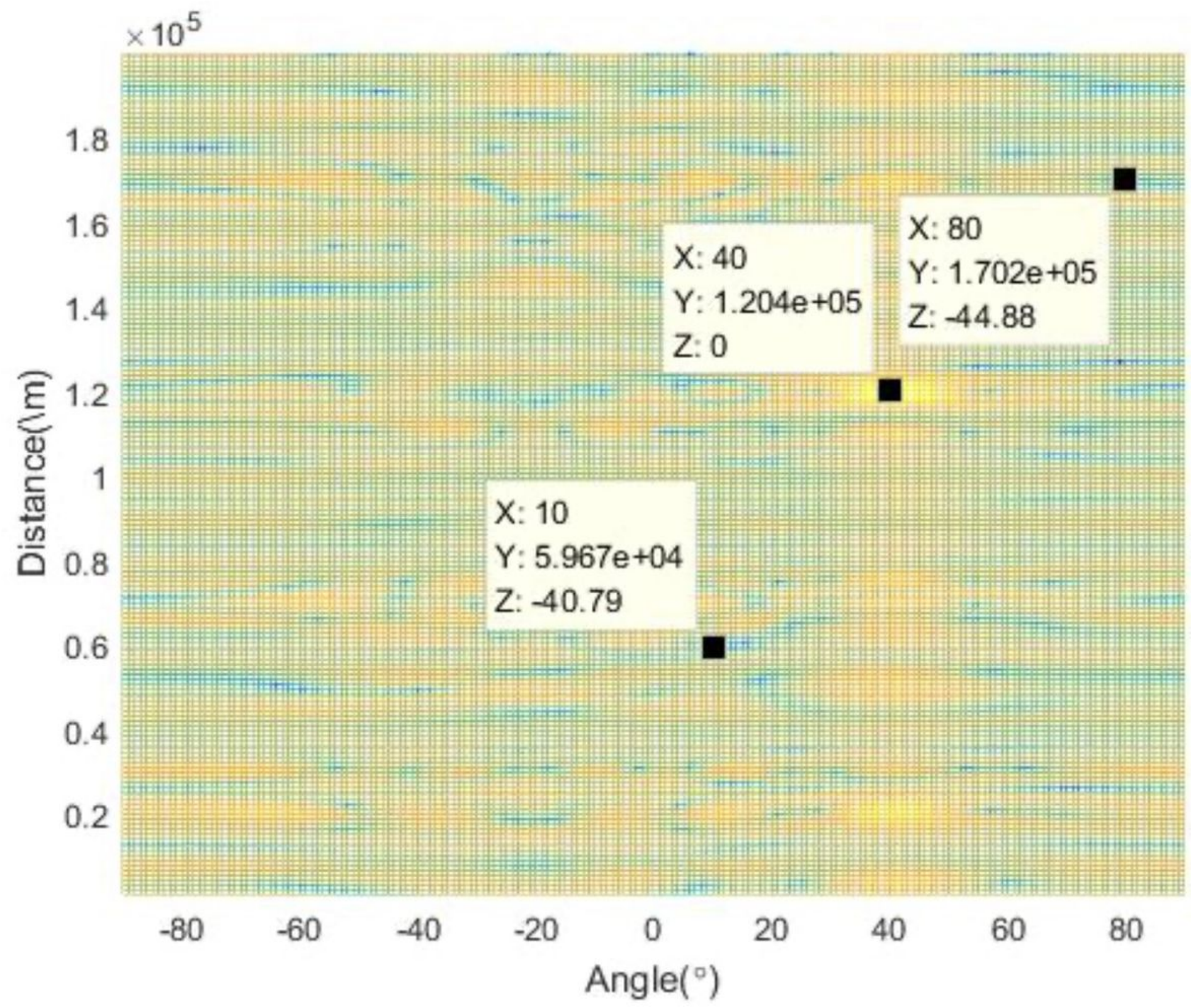

Figure 9

Dot-shape beam of BMP 


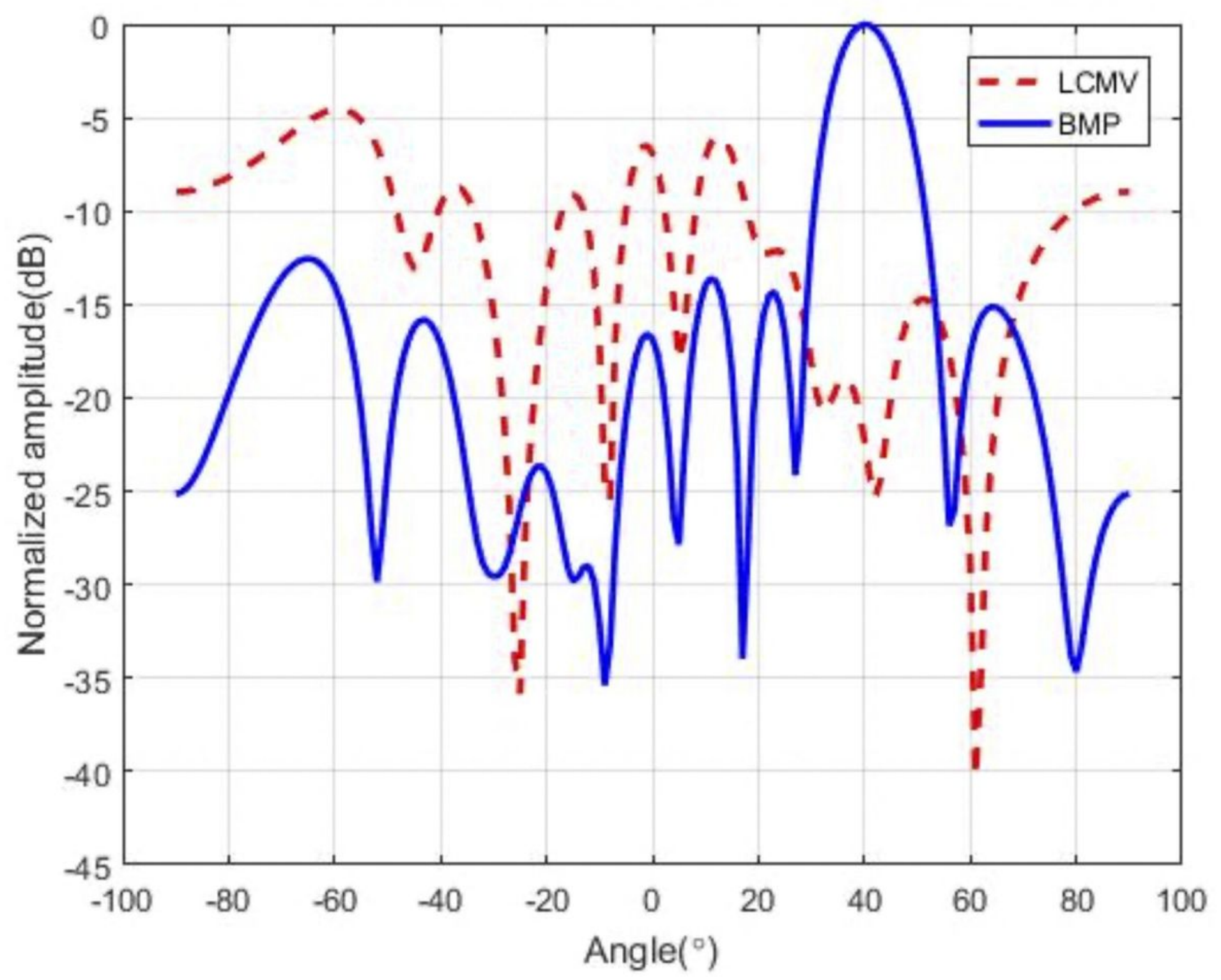

Figure 10

Angle is $40^{\circ}$ 


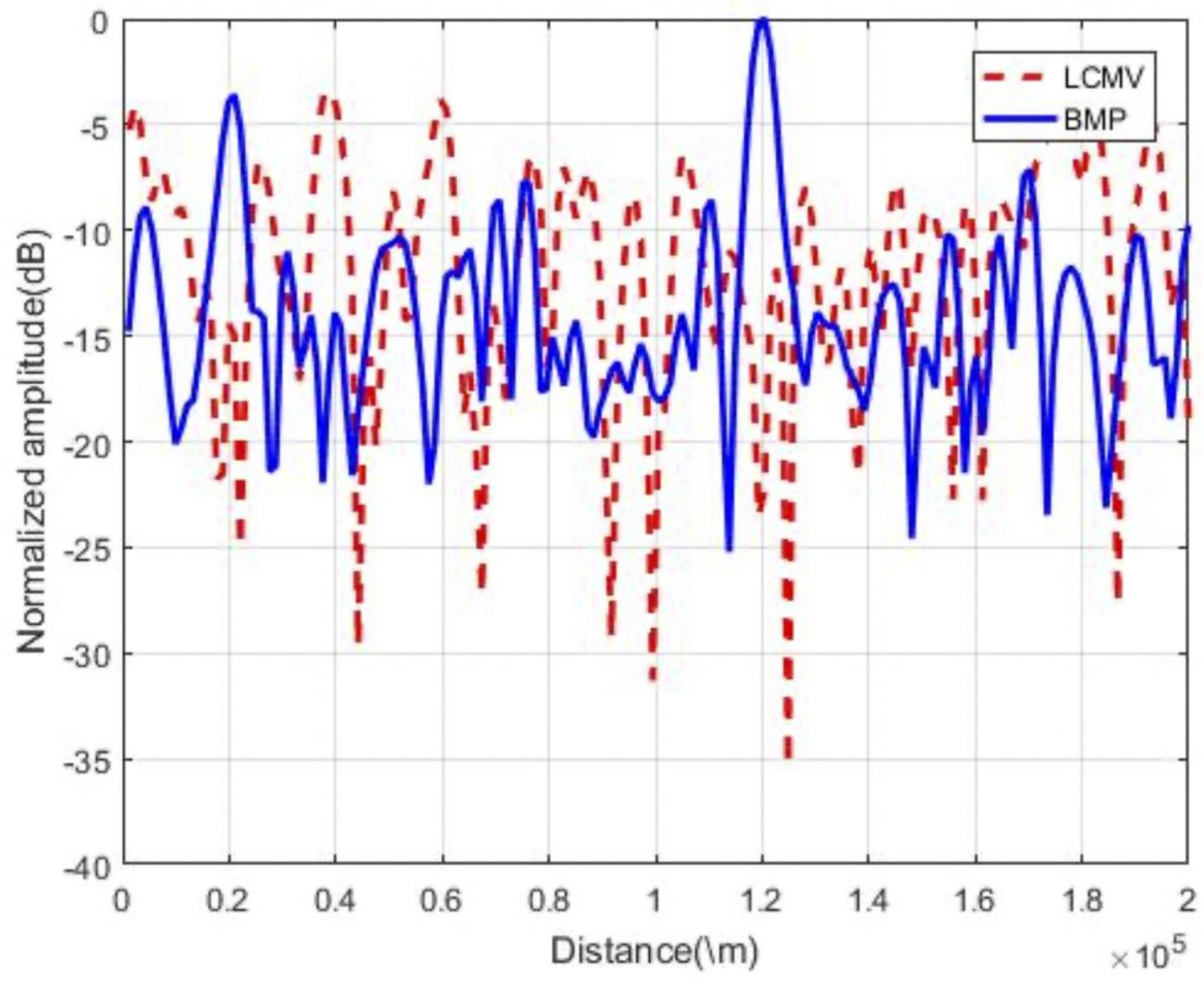

Figure 11

Distance is $120 \mathrm{~km}$ 